\title{
Sensibilidad de biomarcadores en el líquido cefalorraquídeo en enfermedades neurodegenerativas
}

\author{
Guillermo García Ribas 1,5, Isabel Rubio Gómez2,6, José Luis López-Sendón Moreno1,5, \\ María J. Casarejos Fernández ${ }^{2,6}$, Mónica Bascuñana ${ }^{3,5}$ y Justo García de Yébenes ${ }^{4,6}$ \\ ${ }^{1}$ Médico adjunto. ${ }^{2}$ Bióloga molecular. ${ }^{3}$ Neuropsicóloga. ${ }^{4}$ Jefe de sección. \\ ${ }^{5}$ Servicio de Neurología. ${ }^{6}$ Servicio de Neurobiología. \\ Hospital Universitario Ramón y Cajal. Madrid
}

\section{Resumen}

El avance en el conocimiento de la biología de la enfermedad de Alzheimer ha favorecido el desarrollo clínico de biomarcadores que permitan un diagnóstico temprano, monitoricen la progresión y puedan servir parar la evaluación de terapias. Las determinaciones en el líquido cefalorraquídeo (LCR) de beta-amiloide 42 (BA42), proteína tau total y proteína tau fosforilada han mostrado tener un rendimiento diagnóstico elevado para diferenciar la demencia tipo Alzheimer respecto a controles, incluso en fases incipientes de la misma. El rendimiento respecto a otros tipos de enfermedades neurodegenerativas es más bajo, aunque la especificidad de la tau fosforilada se mantiene para diferenciar la demencia tipo Alzheimer de las otras. La aplicabilidad clínica se encuentra limitada por la necesidad de un procedimiento invasivo, y de un protocolo y adiestramiento técnico en la determinación de específicos. La tecnología de medida todavía no está estandarizada a nivel global y la variabilidad entre centros es alta. Sin embargo, en casos clínicos seleccionados y en la investigación de la patocronia de la enfermedad de Alzheimer, los biomarcadores de LCR juegan un papel importante.

(Alzheimer. Real Invest Demenc. 2011;49:26-32)

Palabras clave: Alzheimer, demencia, biomarcadores, péptidos beta-amiloides, proteína tau, líquido cefalorraquídeo.

\section{Abstract}

Recent advances in the pathophysiology of Alzheimer's disease has prompted the clinical development of biomarkers that would expand research in early diagnosis, rate of disease progression and monitoring the effect of therapies. Cerebrospinal fluid measures (CSF) of beta-amyloid 42, total tau and phospo-tau proteins have shown a high diagnostic accuracy in differenciating Alzheimer's dementia from healthy controls, even at very early stages of the disease. Diagnostic accuracy of the biomarkers between Alzheimer's disease and other dementia types is not as good, although the specificity of phospo-tan levels remains high. Clinical usefulness is limited by the invasiveness of the procedure, and by strict adhesion to a well-defined protocol and sample handling. The type of technical method for determination is not widely established, and variability between centres is high. Nevertheless, there is an important role of CSF biomarkers in selected cases, and in the research of timing the events of Alzheimer's disease.

(Alzheimer. Real Invest Demenc. 2011;49:26-32)

Keywords: Alzheimer, dementia, biological markers, amyloid beta-peptides, tau protein, cerebrospinal fluid. 


\section{Introducción}

El síndrome clínico de demencia se caracteriza por la presencia de un deterioro cognitivo, conductual y funcional, generalmente progresivo. Las etiologías vascular y neurodegenerativa cubren la mayoría del espectro diagnóstico y la enfermedad de Alzheimer (EA) es la anatomía patológica predominante dentro de las enfermedades degenerativas, seguida por la demencia con cuerpos de Lewy (dementia with Lewy bodies, DLB) y, a mayor distancia, las degeneraciones lobulares frontotemporales ${ }^{1}$. El diagnóstico de la EA y de otros tipos de enfermedades que se desarrollan con demencia está sustentado en criterios clínicos ${ }^{2}$, cuya sensibilidad es elevada para distinguir entre controles y sujetos afectos. Sin embargo, su especificidad es moderada, por lo que la predicción de la neuropatología basada en la clínica es baja. Las características clínicas se solapan, sobre todo en demencias avanzadas, y además no es infrecuente la coexistencia de distintos procesos patológicos en un mismo paciente ${ }^{3}$.

La presencia de beta-amiloide (BA) en el cerebro humano es un hecho central en la etiopatogenia de la EA, aunque su relación con el desarrollo de la sintomatología clínica no está todavía totalmente aclarada ${ }^{4}$. Es probable que la carga de BA cerebral tenga una relación fisiopatológica precipitante con el resto de las alteraciones neuropatológicas encontradas en la EA, tales como la formación de ovillos neurofibrilares intraneuronales, hilillos del neuropilo, disfunción sináptica, activación de la microglía y cambios vasculares 5 . Sin embargo, esta cascada amiloidogénica todavía se mueve en el terreno de la hipótesis ${ }^{6}$. El inicio de los depósitos de BA en el sistema nervioso ha sido objeto de estudios clínico-patológicos y epidemiológicos que han mostrado, por ejemplo, que en sujetos con síndrome de Down aparecen depósitos décadas antes del deterioro cognitivo que sufren invariablemente ${ }^{7}$. La pre-

Recibido para su publicación: 18 de abril de 2011. Aceptado para su publicación: 2 de junio de 2011. Correspondencia: G. García Ribas.

E-mail: ggarciarib.hrc@salud.madrid.org sencia de depósitos puede estar influida por factores ambientales externos, como se ha demostrado en animales de experimentación sometidos a anestésicos volátiles ${ }^{8}$. También hay placas seniles en cantidad moderada en un $25 \%$ de sujetos cognitivamente intactos en la octava década ${ }^{9}$, lo que equivaldría a la prevalencia clínica de una demencia tipo Alzheimer (DTA) en sujetos mayores de 85 años $^{10}$. Los cambios neuropatológicos en sujetos con criterios clínicos de deterioro cognitivo ligero (DCL) no son uniformes, pero la mayoría presenta una anatomía patológica con depósitos de BA y ovillos neurofibrilares, en línea con los estudios clínicos que sugieren que una gran proporción de sujetos con DCL están en la vía de la DTA ${ }^{11}$. En resumen, los datos epidemiológicos, clínicos y patológicos sugieren que la EA, entendida como el depósito de BA cerebral, puede iniciarse en torno a una década o más antes de las primeras manifestaciones clínicas y una vez que aparecen éstas de forma inequívoca, lo que se ha venido en denominar como DCL, la evolución a demencia suele ocurrir en unos 3-5 años ${ }^{12}$.

Debido a esta dificultad de predicción de la neuropatología por las alteraciones clínicas y a causa de la posibilidad de evidenciar la presencia de anomalías patológicas antes de que el sujeto presente un síndrome de demencia, se han investigado unos biomarcadores que permitan un diagnóstico más específico y, si es posible, más precoz. Un biomarcador se define como una característica que puede medirse de forma objetiva y válida, y que sirve como indicador de un proceso biológico normal o patológico, o una respuesta farmacológica a una intervención terapéutica ${ }^{13}$. En los últimos años, los biomarcadores basados en el líquido cefalorraquídeo (LCR) han demostrado ser una herramienta útil para el manejo diagnóstico de enfermedades neurodegenerativas, sobre todo la EA, incluso antes de que éstas produzcan un síndrome clínico de demencia ${ }^{14}$. Los biomarcadores en el LCR más estudiados son el péptido de 42 aminoácidos de BA, la concentración total de proteína tau y la concentración de proteína tau fosforilada. Sus sensibilidades y especificidades están prácticamente definidas tanto en sujetos con diagnóstico clínico como en estudios con confirmación anatomopatológica. 


\section{Beta-amiloide}

Hace más de dos décadas se comenzaron a determinar las concentraciones de amiloide en el LCR de sujetos con demencia ${ }^{15}$. Los primeros estudios mostraron resultados dispares, sin correlación con la clínica, por la determinación conjunta de toda la fracción soluble de péptidos derivados de la proteólisis de la proteína precursora de amiloide. Posteriormente se pudieron separar distintas formas de BA cortadas en la región C-terminal y demostrar que el péptido más largo de BA, de 42 aminoácidos, tiende a la agregación con más rapidez que los otros y que es el primero que se deposita en las placas seniles (fig. 1). La disminución de su concentración en el LCR se ha correlacionado positivamente con la presencia de alteraciones anatomopatológicas características de la $\mathrm{EA}^{16}$, sobre todo si se relaciona con la concentración del péptido de 40 aminoácidos, que no varía y permite excluir aquellos falsos negativos por hiperproducción de todas las fracciones de BA. Numerosos estudios clínicos han mostrado una sensibilidad que puede situarse en torno al 70-85\% para el diagnóstico de EA clínica con respecto a los controles ${ }^{17}$.

No obstante, el análisis de BA42 en el LCR no es sencillo. El rango esperable de su concentración es muy amplio, de 50 a 1.000 pg/ml, lo que implica utilizar métodos de dilución y control que permitan abarcar este amplio rango ${ }^{18}$. Las concentraciones de BA42 tienen una variación circadiana en el LCR, una vida media corta (2,5-3 min en el plasma) y capacidad para agregarse en materiales de recogida como el poliestireno $^{19}$. De las variables preanalíticas, las que pueden provocar una determinación anormal son el material de recogida, la presencia de un daño de barrera y una

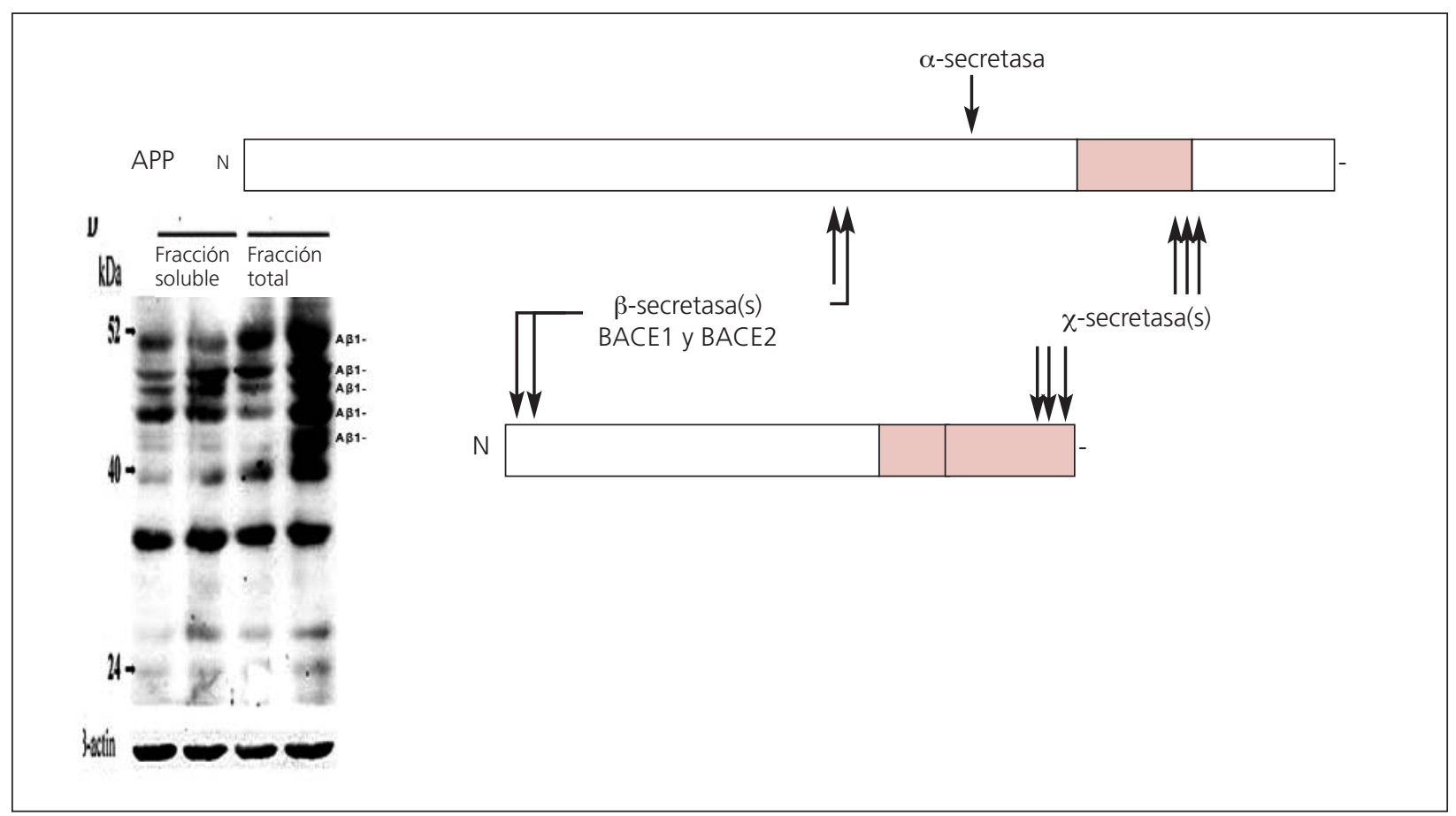

Figura 1. Catabolismo de la proteína precursora de amiloide (APP) por las secretasas. La acción beta-secretasa, seguida por la acción gamma-secretasa, lleva a la constitución de cinco formas de péptidos amiloides (el «quinteto» amiloide). La acción más común de la beta-secretasa rompe la unión metionina-aspártico, siendo éste el primer aminoácido del fragmento beta-amiloide (BA). La acción gamma-secretasa corta la APP por delante de la isoleucina (posición 40) o la de treonina (posición 42), lo que determina la generación de péptidos de distinto peso molecular. También se encuentran péptidos con C-terminal a 37, 38 y 39 residuos, lo que indicaría la presencia de más de una actividad enzimática. Este hecho ha potenciado la búsqueda de dianas terapéuticas para evitar un catabolismo proteico proamiloidogénetico. La zona sombreada indica el dominio transmembrana de la APP. 
centrifugación que provoque una lisis celular ${ }^{20}$. También se han identificado condicionantes analíticos que hacen necesario un protocolo estricto y la validación de los resultados en cada centro ${ }^{21}$.

Los niveles de BA42 se encuentran frecuentemente disminuidos en otras enfermedades neurodegenerativas tales como la DLB ${ }^{22}$, la esclerosis lateral amiotrófica ${ }^{17}$ y en la atrofia multisistema ${ }^{23}$, por lo que su especificidad es relativamente baja. También se encuentran niveles bajos en casos de meningitis bacteriana, de enfermedad de Creutzfeldt-Jakob y de panencefalitis esclerosante subaguda ${ }^{24}$, aunque esto puede ser debido al daño de barrera que se produce. El descenso de BA42 en la EA parece ser un hecho precoz, que refleja un estado cerebral de amiloidosis ${ }^{25}$. Este descenso se mantiene durante todas las fases de la enfermedad, por lo que podría considerarse un marcador de estado: su aparición indicaría una presencia anormal de amiloide, pero sin una correlación clara con la progresión clínica.

\section{Proteína tau total}

La concentración total de proteína tau (tT) se ha utilizado ampliamente como biomarcador de la DTA. Se cree que es un marcador de neurodegeneración, aunque en diversas patologías neurodegenerativas su alteración es mínima ${ }^{26}$. En un meta-análisis reciente ${ }^{27}$, se ha podido constatar la congruencia en la elevación de tT en diversas enfermedades neurodegenerativas respecto a los controles, pero en menor grado que las elevaciones que se producen en pacientes con EA. La concentración de tT se eleva también de forma significativa en la enfermedad de Creutzfeldt-Jakob, y resulta comparable en especificidad a los valores obtenidos con la determinación de la proteína 14-3-328. Una elevación de la proteína tau en sujetos con DCL predice su progresión neuropsicológica, especialmente en aquellos que no presentan alteraciones de señal periventricular extensas. Los niveles de $\mathrm{t} T$ se encuentran transitoriamente elevados tras un ictus agudo y en la demencia vascular. Se ha descrito, en un tercio de los casos con demencia frontotemporal, una disminución de los niveles de tT, un hecho no encontrado en los sujetos con EA29,30.

\section{Proteína tau fosforilada}

La determinación de proteína tau fosforilada (pT), por lo general en el aminoácido 181, añade mayor especificidad al diagnóstico de EA. Su elevación es independiente de la concentración total de proteína tau y los dos biomarcadores no se alteran paralelamente en enfermedades neurodegenerativas. La especificidad de la pT es muy elevada para la EA ${ }^{31}$. Incluso síndromes clínicos que pueden compartir alteraciones anatomopatológicas como la DTA y la DLB pueden distinguirse por la mayor elevación de pT en la DTA ${ }^{22}$. La concentración de pT no se modifica en caso de ictus, depresión mayor, demencia vascular o demencias frontotemporales ${ }^{32}$.

\section{Combinación de biomarcadores}

La combinación de biomarcadores tiene una sensibilidad y especificidad frente a otros tipos de demencia de alrededor del $80-90 \%{ }^{17}$. Los valores predictivos positivos y negativos de la combinación son del 90 y 95\%, respectivamente, y asumen una prevalencia del $45 \% 33$. En un estudio multicéntrico publicado recientemente $\mathrm{e}^{34}$, se llevó a cabo un análisis transversal de pacientes con diagnóstico clínico de EA y controles para determinar los puntos de corte que se utilizarían posteriormente en una muestra prospectiva de sujetos con DCL. Un total de 529 pacientes con EA y 304 controles fueron utilizados para obtener una sensibilidad de los índices del 85\%. Los valores obtenidos fueron $\mathrm{tT}$ $\geq 320 \mathrm{ng} / \mathrm{l}, \mathrm{pT} \geq 52 \mathrm{ng} / \mathrm{l}$ y BA42 $\leq 482 \mathrm{ng} / \mathrm{l}$. Se diseñó una ecuación que combinaba la razón BA42:pT (y) y la $\mathrm{tT}(\mathrm{x})$ para una sensibilidad combinada superior al $85 \%(y=3,694+0,0105 x)$. El uso de esta combinación permite una mayor eficiencia diagnóstica que cada biomarcador por separado, pero deben tenerse ciertas precauciones, ya que la variabilidad entre los centros subraya la necesidad de una estandarización de los procedimientos clínicos y técnicas de análisis. Se ha propuesto que la concentración de BA42 representa el estado de patología Alzheimer, mientras que tT y pT serían marcadores de intensidad, en los que unos nive- 
les más elevados indicarían una progresión clinica más rápida ${ }^{25}$ (fig. 2).

\section{Redefiniendo la enfermedad de Alzheimer}

La buena eficiencia diagnóstica de los biomarcadores en las fases tempranas de la DTA ha hecho que se esté estudiando su rentabilidad en el DCL, en las quejas subjetivas de memoria y en la población asintomática anciana. De esta forma, se abre un nuevo glosario de términos para definir el espectro de la EA (tabla 1$)^{35}$. La EA se definiría como una enfermedad bifásica, con una fase asintomática y alteraciones anatomopatológicas silentes, de duración por lo general muy larga (¿décadas?) y una fase sintomática inicialmente paucisintomática y, posteriormente, de demencia. La fase sintomática inicial, denominada prodrómica, cumpliría con los criterios que definen el DCL. La alteración de la memoria episódica, objetivada mediante test neuropsicológicos y asociada a una alteración en los biomarcadores, definiría al sujeto como enfermo de Alzheimer sin necesidad de estar demente. Aquellos pacientes que se encuentren en el rango de demencia tendrán una EA típica, si hay afectación de memoria, de praxias y de orientación, o EA atípica, si se alteran otras funciones cognitivas. El término asintomático con riesgo de EA se ha propuesto para definir el estado de sujetos sin alteraciones clínicas que presentan una positividad de biomarcadores sugestivos de patología amiloide. Finalmente, el término enfermedad de Alzheimer asintomática se reserva para aquellos sujetos sin síntomas pero portadores de una mutación monogénica conocida como causa de Alzheimer autosómico dominante.

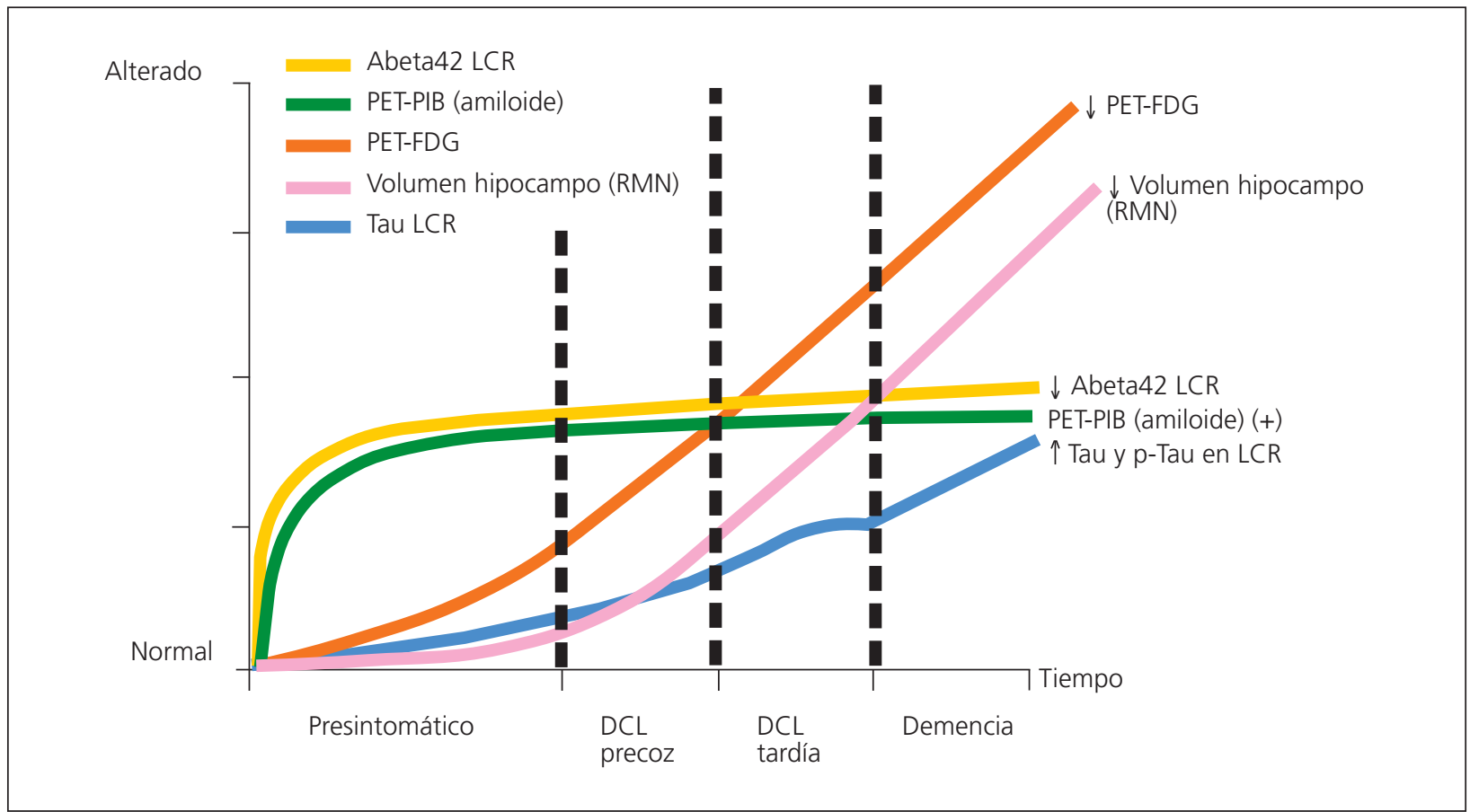

Figura 2. Biomarcadores y progresión de la enfermedad de Alzheimer. La disminución del beta-amiloide 42 (BA42) en el líquido cefalorraquídeo (LCR) y el ligamiento al amiloide fibrilar por el compuesto B de Pittsburgh (PIB) analizado mediante la tomografía por emisión de positrones (PET) serían detectables en fases de la enfermedad patológica, aún sin síntomas clínicos, pero apenas se modificarían con el curso clínico. La alteración en las concentraciones de tT y pT indicarían neurodegeneración y progresión clínica. La volumetría del hipocampo y la captación de glucosa (FDG) serían también marcadores de progresión clínica, incrementando su alteración con la evolución del cuadro clínico.

DCL: deterioro cognitivo ligero; RMN: resonancia magnética nuclear. 


\begin{tabular}{|l|l|l|l|l|}
\hline Denominación & $\begin{array}{l}\text { Dx } \\
\text { EA }\end{array}$ & $\begin{array}{l}\text { Alteración en } \\
\text { memoria episódica }\end{array}$ & Biomarcadores & Requisitos adicionales \\
\hline Demencia por EA & Sí & Obligada & Sí & Presencia de demencia \\
\hline EA típica & Sí & Obligada & Sí & Ninguno \\
\hline EA atípica & Sí & No necesaria & Sí & Presentación clínica específica \\
\hline EA prodrómica o estadio predemencia & Sí & Obligada & Sí & Ausencia de demencia \\
\hline EA mixta & Sí & Obligada & Sí & Evidencia de comorbilidad \\
\hline Situación preclínica con riesgo de EA & No & No está presente & Sí & Ausencia de síntomas \\
\hline EA presintomática & No & No está presente & No necesarios & $\begin{array}{l}\text { Ausencia de síntomas } \\
\text { y mutación monogénica }\end{array}$ \\
\hline Deterioro cognitivo ligero & No & No necesaria & No necesarios & $\begin{array}{l}\text { Ausencia de síntomas o } \\
\text { biomarcadores }\end{array}$ \\
\hline
\end{tabular}

Tabla 1. Definiciones utilizadas en la enfermedad de Alzheimer clínica.

EA: enfermedad de Alzheimer.

\section{Conclusiones}

Estamos asistiendo a cambios en los paradigmas de diagnóstico de las enfermedades neurodegenerativas. Los primeros vinieron de la mano de la genética, concretamente del descubrimiento de la alteración genética responsable de la enfermedad de Huntington. La expansión de tripletes en uno de los alelos del gen que codifica la huntingtina provoca la mutación de ésta y es causa necesaria y suficiente para el desarrollo de los síntomas clínicos. Pero estos avances en medicina predictiva se han frenado en enfermedades donde la aparición de síntomas no se debe tan sólo a la presencia de una alteración anatomopatológica. Queda también por dilucidar la intervención del envejecimiento en la neurodegeneración. Un primer paso está siendo la delimitación de biomarcadores, que permitirán sobre todo su aplicación para refinar los ensayos clínicos y permitir un mejor ajuste de los tratamientos de este grupo de enfermedades. Por el momento, los biomarcadores más ampliamente utilizados para la EA no deben ser aplicados de forma rutinaria en la práctica clínica, ya que no permiten un diagnóstico aisladamente. Sin embargo, demuestran su utilidad en casos atípicos, y como ayuda de la neuropsicología y las pruebas de imagen.

\section{Bibliografía}

1. López-Pousa S, Garre-Olmo J. Las demencias: concepto y epidemiología. En: Alberca R, López-Pousa S (eds.). Enfermedad de Alzheimer y otras demencias, 4. ${ }^{\mathrm{a}}$ ed. Madrid: Médica Panamericana; 2010. pp. 29-40.

2. Knopman DS, DeKosky ST, Cummings JL, Chui H, Corey-Bloom J, Relkin N, et al. Practice parameter: diagnosis of dementia (an evidence-based review). Report of the Quality Standards Subcommittee of the American Academy of Neurology. Neurology. 2001;56:1 143-53.

3. Duyckaerts C. Neuropathologic classification of dementias: introduction. Handb Clin Neurol. 2008;89:147-59.

4. Prohovnik I, Perl DP, Davis KL, Libow L, Lesser G, Haroutunian V. Dissociation of neuropathology from severity of dementia in late-onset Alzheimer disease. Neurology. 2006;66:49-55.

5. Mena MA, Rodríguez-Navarro JA, De Yébenes JG. The multiple mechanisms of amyloid deposition: the role of parkin. Prion. 2009;3:5-11.

6. Mondragón-Rodriguez S, Basurto-Islas G, Lee H, Perry G, Zhu X, Castellani RJ, et al. Causes versus effects: the increasing complexities of Alzheimer's disease pathogenesis. Expert Rev Neurother. 2010;10:683-91.

7. Rumble B, Retallack R, Hilbich C, Simms G, Multhaup G, Martins R, et al. Amyloid A4 protein and its precursor in Down's syndrome and Alzheimer's disease. N Engl J Med. 1989;320:1446-52.

8. Perucho J, Rubio I, Casarejos MJ, Gomez A, RodriguezNavarro JA, Solano RM, et al. Anesthesia with isoflurane increases amyloid pathology in mice models of Alzheimer's disease. J Alzheimers Dis. 2010;19:1-13.

9. Davis DG, Schmitt FA, Wekstein DR, Markesbery WR. Alzheimer neuropathologic alterations in aged cognitively 
normal subjects. J Neuropathol Exp Neurol. 1999;58: 376-8.

10. Ferri CP, Prince M, Brayne C, Brodaty H, Fratiglioni L, Ganguli M, et al. Global prevalence of dementia: a Delphi consensus study. Lancet. 2005;366:2112-7.

11. Markesbery WR. Neuropathologic alterations in mild cognitive impairment: A Review. J Alzheimers Dis. 2010;19: 221-8.

12. Davatzikos C, Xu F, An Y, Fan Y, Resnick SM. Longitudinal progression of Alzheimer's-like patterns of atrophy in normal older adults: the SPARE-AD index. Brain. 2009; 132: 2026-35.

13. Naylor S. Biomarkers: current perspectives and future prospects. Expert Rev Mol Diagn. 2003;3:525-9.

14. Blennow K, Hampel H. CSF markers for incipient Alzheimer's disease. Lancet Neurol. 2003;2:605-13.

15. Palmert MR, Podlisny MB, Witker DS, Oltersdorf T, Younkin LH, Selkoe DJ, et al. The beta-amyloid protein precursor of Alzheimer disease has soluble derivatives found in human brain and cerebrospinal fluid. Proc Natl Acad Sci USA. 1989;86:6338-42.

16. Tapiola T, Alafuzoff I, Herukka S-K, Parkkinen L, Hartikainen $\mathrm{P}$, Soininen $\mathrm{H}$, et al. Cerebrospinal fluid ,-amyloid 42 and tau proteins as biomarkers of Alzheimer-type pathologic changes in the brain. Ann Neurol. 2009; 66: 382-9.

17. Blennow $\mathrm{K}$, Hampel $\mathrm{H}$, Weiner $\mathrm{M}$, Zetterberg $\mathrm{H}$. Cerebrospinal fluid and plasma biomarkers in Alzheimer disease. Nat Rev Neurol. 2010;6:131-4.

18. Verwey NA, Van der Flier WM, Blennow K, Clark C, Sokolow S, De Deyn PP, et al. A worldwide multicentre comparison of assays for cerebrospinal fluid biomarkers in Alzheimer's disease. Ann Clin Biochem. 2009;46:235-40.

19. Ghiso J, Shayo M, Calero M, Ng D, Tomidokoro Y, Gandy S, et al. Systemic catabolism of Alzheimer's Abeta40 and Abeta42. J Biol Chem 2004;279:45897-908.

20. Bjerke M, Portelius E, Minthon L, Wallin A, Anckarsäter $\mathrm{H}$, Anckarsäter R, et al. Confounding factors influencing amyloid beta concentrations in cerebrospinal fluid. Int J Alzheimers Dis. 2010 Jul 15;2010. pii: 986310.

21. Mattsson N, Blennow K, Zetterberg H. Inter-laboratory variation in cerebrospinal fluid biomarkers for Alzheimer's disease: united we stand, divided we fall. Clin Chem Lab Med. 2010;48:603-7.

22. Gómez-Tortosa E, Gonzalo I, Fanjul S, Sainz MJ, Cantarero S, Cemillán C, et al. Cerebrospinal fluid markers in dementia with Lewy bodies compared with Alzheimer disease. Arch Neurol. 2003;60:1218-22.

23. Holmberg B, Johnels B, Blennow K, Rosengren L. Cerebrospinal fluid A,42 is reduced in multiple system atrophy but normal in Parkinson's disease and progressive supranuclear palsy. Movement Disorders. 2003;18: 186-90.

24. Lewczuck P, Wiltfang J. Neurochemical dementia diagnostics: State of the art and research perspectives. Proteomics. 2008;8:1292-301.

25. Jack CR, Knopman DS, Jagust WJ, Shaw LM, Aisen PS, Weiner MW, et al. Hypothetical model of dynamic biomarkers of the Alzheimer's pathological cascade. Lancet Neurol. 2010;9:1 19-28.

26. Ballatore C, Lee VM, Trojanowski JQ. Tau-mediated neurodegeneration in Alzheimer's disease and related disorders. Nat Rev Neurosci. 2007;8:663-72.

27. Van Harten AC, Kester MI, Visser P-J, Blankenstein MA, Pijnenburg YAL, Van der Flier WM, et al. Tau and p-tau as CSF biomarkers in dementia: a meta-analysis. Clin Cehm Lab Med. 2011;49:353-66.

28. Otto M, Wiltfang J, Cepek L, Neumann M, Mollenhauer B, Steinacker P, et al. Tau protein and 14-3-3 protein in the differential diagnosis of Creutzfeldt-Jakob disease. Neurology. 2002 Jan 22;58(2):192-7.

29. Maruyama M, Matsui T, Tanji H, Nemoto M, Tomita N, Ootsuki M, et al. Cerebrospinal fluid tau protein and periventricular white matter lesions in patients with mild cognitive impairment: implications for 2 major pathways. Arch Neurol. 2004;61:716-20.

30. Grossman M, Farmer J, Leigh S, Work M, Moore P, Van Deerlin V, et al. Cerebrospinal fluid profile in frontotemporal dementia and Alzheimer's disease. Ann Neurol. 2005;57:721-9.

31. Monje-Argilés JA, Sánchez-Payá J, Muñoz-Ruiz C, Pampliega-Pérez A, Montoya-Gutiérrez J, Leiva-Santana C. Biomarcadores en el líquido cefalorraquídeo de pacientes con deterioro cognitivo leve: metaanálisis de su capacidad predictiva para el diagnóstico de la enfermedad de Alzheimer. Rev Neurol. 2010; 50:193-200.

32. Schipper H. Biological markers and Alzheimer disease: a Canadian perspective. Int J Alzheimers Dis. 2010 Aug 8; 2010. pii:978182.

33. Shoji M. Cerebrospinal fluid Abeta40 and Abeta42: natural course and clinical usefulness. Front Biosci. 2002;7: 997-1006.

34. Mattsson N, Zetterberg H, Hansson O, Andreasen N, Parnetti L, Jonsson M, et al. CSF biomarkers and incipient Alzheimer disease in patients with mild cognitive impairment. JAMA. 2009;302:385-93

35. Dubois B, Feldman HH, Jacova C, Cummings JL, de Kosky ST, Barberger-Gateau P, et al. Revising the definition of Alzheimer's disease: a new lexicon. Lancet Neurol. 2010;9:1118-27. 